Fernando Blaya

Universidad Politécnica de Madrid, Spain

Silvia Nuere

Universidad Politécnica de Madrid, Spain

Manuel IsLÁN

Universidad Politécnica de Madrid, Spain

\title{
ART AND SCIENCE: 3D TECHNOLOGIES AT THE SERVICE OF CULTURE
}

Tell me and I forget, teach me and I remember, involve me and I learn.

Benjamin Franklin

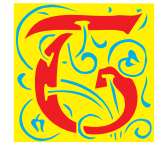

he European Higher Education Area (EHEA) was launch to ensure more comparable, compatible and coherent systems of higher education in Europe. This scenario has developed new methodologies based on competences and force professors to redefine the way of teaching. One major change is related to focus on the formative processes, more specifically expressed in the results of learning in terms of competences.

The EHEA has defined common guidelines for the European universities to increase concepts related to the student learning process and with a bigger interest focus on investigation. All in all, we look for a learning-connection between the professional world and lifelong learning.

Martínez-Otero (García-Alonso 2016), in 1997, has already told us some of the problems we have in higher education and especially in engineering. 
There is an excess of theoretical content of subjects and a lack of practice and experience in real contexts.

Therefore we need to find a methodology based on a teaching-learning process that could better prepare our students in competences, knowledge, general skills and attitudes.

If we take into consideration the White Paper of the European Commission "Teaching and learning. Towards the society of knowledge" (Riesco 1995) we will be interested in two headings:

- Encourage the acquisition of new knowledge (avoid obsolescence).

- Bring the university to the business world.

This brings us the possibility of working through projects and with real needs of the society.

But we are not only interested in teaching engineering, and as a group of professors, coming from different professional training, we think that combine all the knowledge coming from apparently such a different areas (art, engineering) can bring a better opportunity to learn.

Society is a mix of disciplines and an opportunity to bring new scenarios to our students. If we think in a wide range about European culture, Spain with its cultural legacy is a perfect opportunity to make a proposal of investigation and transfer it into university, so as to improve, not only the way of teaching, but also to show that a real project can be integrated into our classes without forgetting new technologies within our reach.

We then talk about teaching based on projects and here we have an example with the digitalization of the wine cellars in Aranda de Duero, Spain.

\section{Project}

A good illustration of the uses of 3D technology is the digitalisation of a set of underground cellars located in Aranda de Duero (Spain) which was declared an Asset of Cultural Interest in 2013 by the Government of Spain. Some students could work in this project and experience a real possibility to transfer the theoretical classes into a real proposal.

\section{Aranda de Duero}

First of all, we have to tell that the present group of cellars in Aranda de Duero is, from an historic and ethnographic point of view, the main cause of the 
development of the city. In fact, the increase of the area of this town is closely produced in a parallel way as the construction of the wine cellars built in the underground (Anexo BOE No 175/ jueves 23 de julio de 2015. Sec. III. p. 62237).

The underground of Aranda de Duero has around 135 cellars dating approximately before the XVI century. There has been a deep study of 60 of them, taking photos and scanning them in order to understand the space and its functionality. We also have to mention that a lot of them are more or less abandoned, others are private and just one of them is for tourist visit.

This kind of study made with the use of 3D technologies allows us to promote and take care of a wonderful heritage by creating an inventory of them. It has been created a virtual video tour to visit all of them so you can have an immersion without the necessity of being there.

\section{Methodology}

To take into consideration the wine cellars of Aranda, we started from aspects as investigation of previous information, photographs, maps and book reviews, so as to know, from a true aspect, the characteristics of the object of study.

We decided to approach it from an architectural-archaeology point of view and using reverse engineering. Such tools make necessary to work on the site, and here we can appreciate the importance of teaching through project based learning. It is obviously different to experiment it than to hear a master class about it.

Thanks to new technologies of digitalization we can create a virtual catalogue of the cultural heritage. Current software will allow us, once we take all the photos, to restructure the object in three dimensions for later use. Utilizing the scanner and photogrammetry we will obtain the three dimensions of the wine cellar spaces with an excellent definition. Once we have done all this process we can reproduce them through $3 \mathrm{D}$ printers, promotional or tourist videos to watch them in a digital platform.

We will explain, in a summarising way, the tools we have used to make this investigation come true.

\section{Laser scanner}

With the scanner we can take easily the form, texture and colour of different objects, buildings or landscapes due to the sum of the laser rays and the 
subsequent measurement of the distance in each of the directions we point to. After all, we will be able to join all the information and transform it into $3 \mathrm{D}$ images with the use of specific software.

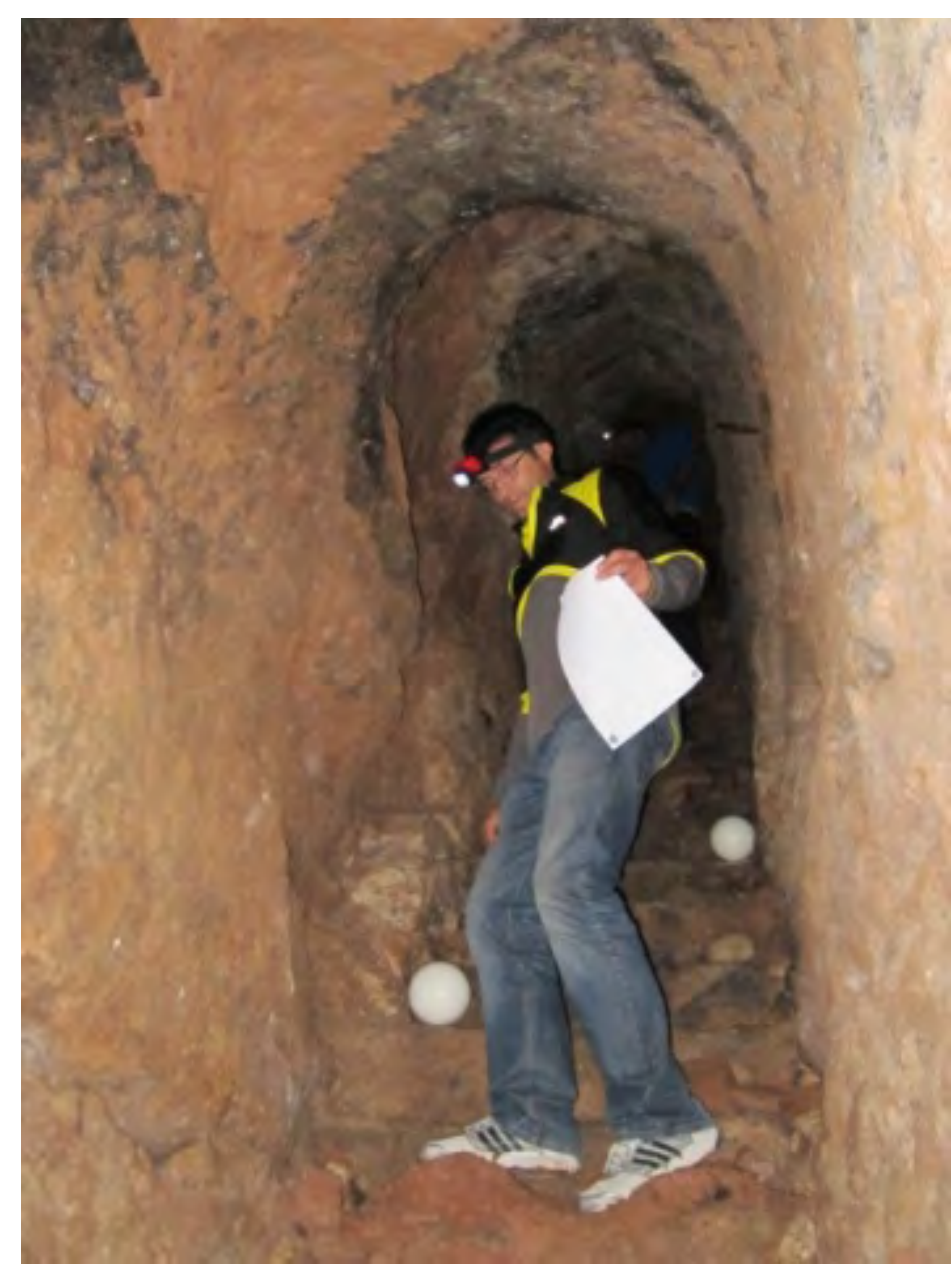

Figure 1. Preparation of the scan and reference points (white spheres)

Source: Fernando Blaya’s photograph.

\section{Panoramic photography}

We will define the panoramic photography as the one that has the same aspect relation that perceives the human eye with a proportion of $2: 1$. We can also include the environment of the shoot of the camera, which is $360^{\circ}$ in the horizontal axis and $180^{\circ}$ in the vertical axis. 


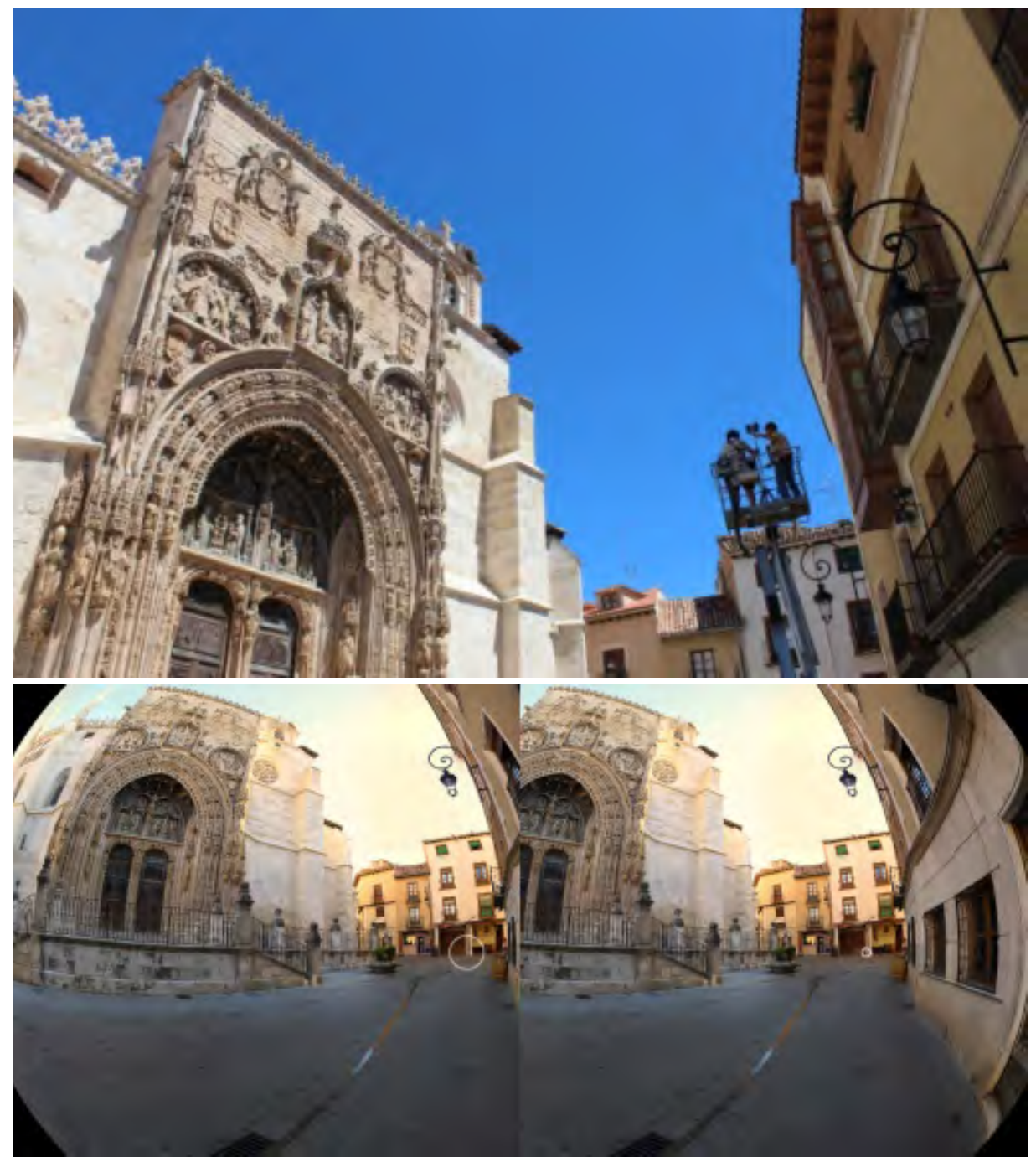

Figure 2. Shooting of the façade of the church of Santa Maria and vision with Oculus

Source: Fernando Blaya’s photograph.

\section{Photogrammetry}

Photogrammetry is a technique that allows three-dimensional photograph restitution from geometry of the shape and dimensions and its texture or material aspect. Depending on the quality we want to have, we will need a different quantity of photographs. 


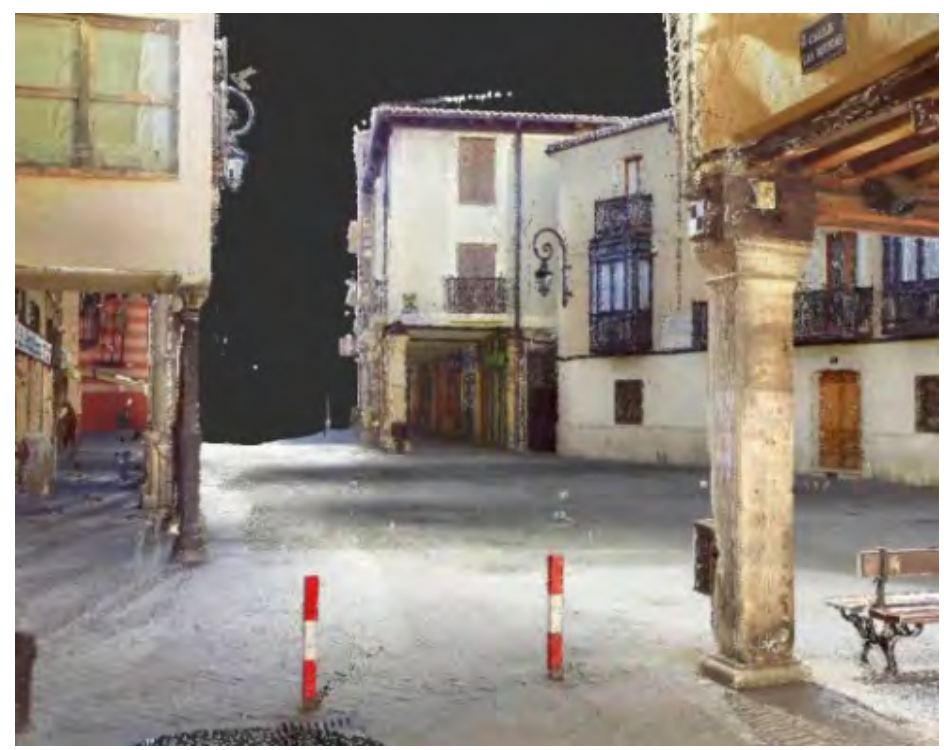

Figure 3. View of the Trigo Square

Source: Fernando Blaya’s photograph.

The aim is the complete restitution of the space thanks to photos.

There is different software that processes all the pictures taken and aligns them to recompose the photographed object in three dimensions. Once we have edited we can texture it and obtain ortho-photos.

\section{Video and panoramic video}

Creating a video is a key objective in the process. It will serve to allow the location of the referenced object in space and it will work, as well, as an important resource for the development and promotion of cultural heritage.

If we focus on aspects related to tourism, the video is really interesting for its ability to recreate itineraries that simulate a real journey, which would display the heritage filmed at any time and from anywhere.

The process to generate a panoramic video is similar to the one we describe in the panoramic photograph process. In this case we will use a six video cameras mounted on a stand with a cube configuration to cover the whole environment. As we need that all the cameras start at the same moment, they will be synchronized. A video will be generated as a result of the cylindrical projection of the various video sources placed in a spherical space. 


\section{Planimetry. Floor plan. 2 dimension computer graphics}

In spite of the fact that the characteristics of the wine cellars are irregular, thanks to the digitalization we could obtain precise floor plan. With a specific computer tool, made for this occasion to generate digital topographic models, we create outlines that became the shapes where later on we included the photographic image related to each zone with all the texture, colour and definition.
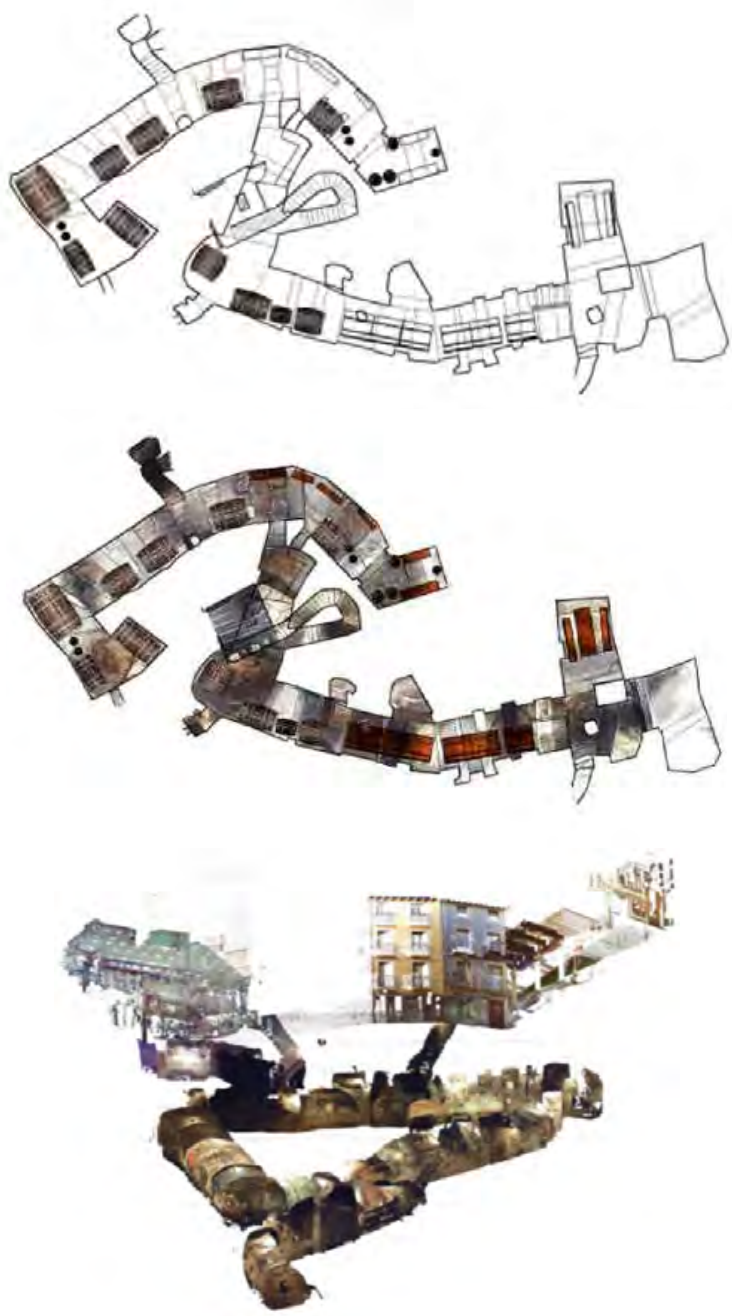

Figure 4a, 4b \& 4c. Outlines extracted from the drawing software. Superimposed image. 3D computer graphics in high quality

Source: Fernando Blaya. 
On the one hand, technologies currently available are virtually essential for the study, preservation and dissemination of cultural heritage, being totally recommended it use and promotion in the practice of numerous disciplines such as archaeology, inventoried goods, or tourism promotion, among others.

The possibility of creating 3D models after scanning can be use in two ways:

- For the creation of a 3D inventory that could be use in a virtual way and as reverse engineering to recuperate damaged or disappeared goods and,

- To create models used for educational interest (blind person, in a class of Art history or Geography, Design engineering, etc).

Thanks to new digital technologies, especially the internet and 3D printing, Europe's rich cultural heritage can today reach new audiences across the world. They can be used to create virtual catalogues of the entire range of European cultural history, from buildings and urban settings to artefacts.

On the other hand, we consider that learning based on projects is an adequate proposal in this educational frame, not only because it can be well adapted into it, achieving their requirements, but also because the transversal competences, as collaborative work or interdisciplinary coordination, can be developed. Ours students of industrial engineering design may study the problems framed into a real project.

Our investigation is also based on reverse engineering and on teaching methodologies in training for innovation, making a proposal of activity related with the Spanish cultural legacy, with art and engineering. This kind of proposal should be implement in the curriculum of some engineering programs, in particular in the degree in industrial design and product development.

Reverse engineering is, in turn, an excellent tool for innovation and a teaching strategy to acquire the skills required for design and innovation in engineering education (Wanamaker 2012).

The study methodology presented here is to propose reverse engineering as a novel technique that not only discloses the historical, artistic and cultural heritage, but it also encourages the preservation of its incalculable wealth.

Finally, it could be added as additional conclusion that even though we are a group of professors from the Polytechnic University of Madrid trained in such different areas as Fine Arts and Industrial Engineering we find that work, mixing different fields, provides students a wide insight into their training. Is it necessary then to come back to a humanism training, muddling up all disciplines? 


\section{References}

Acuerdo 40/2015, de 25 de junio, de la Junta de Castilla y León, por el que se declaran las Bodegas de Aranda de Duero (Burgos), bien de interés cultural con categoría de conjunto etnológico (2015).

Blaya-Haro, F., Estudio integral del conjunto de bodegas históricas de la localidad de Aranda de Duero, Madrid: URJC (Tesis inédita), 2014.

García-Alonso, J.M., Aplicación de una metodología didáctica a estudiantes de ingeniería mecánica para mejorar su empleabilidad e inserción laboral, Badajoz: UEX (Tesis inédita), 2016.

Wanamaker, T., \& Miller, C., Implementing reverse Engineering Methodology into First Year Engineering Curricula from a Student Perspective, 66th EDGED mid-Year Conference Proceedings (p. 133), Galveston, Texas: Purdue University, 2012. 